\title{
Depressão resistente a tratamento: uma revisão das estratégias farmacológicas de potencialização de antidepressivos
}

\author{
Treatment-resistant depression: review of pharmacologic antidepressant \\ strategies
}

\author{
Milena Antunes Santos, Cláudia Hara, Bárbara Luciane Perdigão Stumpf e \\ Fábio Lopes Rocha
}

\begin{abstract}
Resumo
Objetivo: Fazer uma revisão sobre oito estratégias farmacológicas de potencialização de antidepressivos na DRT. Métodos: Fez-se um levantamento bibliográfico de 1990 até janeiro de 2006, nas bases eletrônicas de busca Medline, LILACS e da Biblioteca Cochrane, utilizando-se os termos de busca treatment, resistant, refractory e depression e os descritores depression, drug resistance e augmentation, incluindo apenas ensaios controlados duplo-cegos. Foi consultada a referência dos artigos para obtenção de ensaios realizados em data anterior a 1990 e artigos originais de valor histórico. Resultados: Foram encontrados 17 estudos duplo-cegos com o lítio, seis com o hormônio tireoidiano, dois com a buspirona, seis com o pindolol, um com a carbamazepina, dois com a lamotrigina e quatro com a olanzapina. Foram favoráveis à potencialização $41,2 \%$ dos ensaios com lítio; $60 \%$ daqueles com hormônio tireoidiano e antidepressivos tricíclicos e nenhum com hormônio tireoidiano e inibidores seletivos da recaptação da serotonina (ISRS); $50 \%$ dos com pindolol; $100 \%$ dos ensaios com carbamazepina e $40 \%$ daqueles com olanzapina. Nenhum dos estudos com a buspirona foi favorável. No único estudo com lamotrigina não houve eficácia de tratamento na avaliação pelo critério principal, mas superioridade ao placebo em critérios secundários. Conclusão: Na DRT há evidência de eficácia apenas em relação ao lítio na potencialização de várias classes de antidepressivos e ao hormônio tireoidiano na potencialização de tricíclicos. A olanzapina foi razoavelmente estudada e sua eficácia não foi estabelecida. Os poucos estudos realizados com a buspirona e 0 pindolol não comprovaram sua eficácia. A carbamazepina foi muito pouco estudada, e a lamotrigina ainda não foi adequadamente avaliada.

Palavras-chave: depressão, resistência a drogas, potencialização.
\end{abstract}

\begin{abstract}
Objective: The aim of this study is to review eight pharmacologic antidepressant augmentation strategies in TRD. Methods: Database search on Medline, LILACS and Cochrane Library, from 1990 to June 2006 using the words treatment, resistant, refractory, depression and the medical subject headings depression, drug resistance and augmentation. Double-blind controlled trials and reviews were included. We also consulted reference of the articles in order to obtain studies and original articles of historical value from before 1990. Results: There were 17 double-blind trials with lithium, six with thyroid hormone, two with buspirone, six with pindolol one with carbamazepine, two with lamotrigine and four with olanzapine. Forty-one percent of the trials with lithium, $60 \%$ of those with thyroid hormone and tricyclics, $0 \%$ of the ones with thyroid hormone and selective serotonin reuptake inhibitors (SSRI), $50 \%$ of those with pindolol, $100 \%$ of those with carbamazepine and $40 \%$ of the ones with olanzapine were favorable. No trials with buspirone were favorable. The only trial with lamotrigine did not show efficacy using the main outcome measures. Otherwise, there was superiority over placebo on secondary measures. Conclusion: Only lithium and thyroid hormone showed efficacy as antidepressant augmentation strategies for TRD. Olanzapine was reasonably studied and did not prove its efficacy. There were just a few studies on buspirone and pindolol and they were not favorable to them. Carbamazepine was studied very little. Lamotrigine was not adequately evaluated.
\end{abstract}

Key words: depression, drug resistance, augmentation.

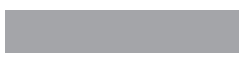

Instituto de Previdência dos Servidores do Estado de Minas Gerais (IPSEMG) (Santos MA, Stumpf BLP)

Faculdade de Medicina da Universidade Federal de Minas Gerais (FM/U) (Hara C)

Recebido

22-06-06

Aprovado Universidade de Brasília (UnB) (Rocha FL)

$11-09-06$

Trabalho realizado no IPSEMG.

Correspondência para: Milena Antunes Santos

Rua Domingos Vieira, 587/1110 - Santa Efigênia - 30150-240 - Belo Horizonte-MG -

Tel.: (31) 3241-4668/9979-0129 - e-mail: milena73@yahoo.com
} 


\section{Introdução}

A depressão é uma doença freqüente, incapacitante, crônica e onerosa (Greenberg et al., 2004). Cerca de 50\% dos pacientes que iniciam tratamento para depressão não respondem à primeira medicação antidepressiva (Thase, 2004); $29 \%$ a $46 \%$ deles não se beneficiam de sucessivos ensaios terapêuticos (Fava e Davidson, 1996; Fava, 2001). Resposta inadequada ou ausência de resposta a um ou mais antidepressivos define a depressão resistente a tratamento (DRT). Mesmo com os esforços para a realização de ensaios clínicos, esses ainda não são suficientes ou significativos para o estabelecimento da superioridade entre estratégias para a terapêutica da DRT ou para a proposição de um consenso ou algoritmo de tratamento.

As estratégias terapêuticas mais freqüentemente utilizadas na DRT são substituição de antidepressivo, combinação e potencialização de antidepressivos. A potencialização é definida como a adição de um segundo agente farmacológico, não-antidepressivo, ao antidepressivo em uso, com o objetivo de atingir a remissão da sintomatologia depressiva (Thase et al., 1998). É indicada em casos de resposta parcial há pelo menos quatro semanas de uso do antidepressivo em dose terapêutica, seguidas de mais duas semanas na dose máxima tolerada (Thase et al., 1998).

A principal vantagem da potencialização é não requerer a suspensão do antidepressivo em uso, ou seja, não se perderem os benefícios já adquiridos (Thase et al., 1998; Bailey, 2003; Thase, 2004). Outras vantagens seriam a possibilidade de resposta mais rápida e a redução da dose de uma ou de ambas as drogas, minimizando seus efeitos colaterais (Sussman e Joffe, 1998; Thase et al., 1998). Entretanto, existem desvantagens na potencialização, como a possibilidade de interação medicamentosa prejudicial, efeitos adversos adicionais e pior adesão ao tratamento (Thase et al., 1998).

Neste trabalho, far-se-á uma revisão dos estudos envolvendo sete fármacos utilizados na potencialização de antidepressivos: lítio, hormônio tireoidiano, buspirona, pindolol, carbamazepina, lamotrigina e olanzapina na DRT.

\section{Métodos}

Fez-se uma revisão da literatura a partir de 1990 até janeiro de 2006, nas fontes eletrônicas de busca Medline, LILACS e da Biblioteca Cochrane. As estratégias de busca dos artigos no Medline e no LILACS basearam-se nos termos de busca treatment, resistant, refractory e depression e nos descritores depression, drug resistance e augmentation. $\mathrm{Na}$ Biblioteca Cochrane, buscaram-se artigos de revisão sistemática e ensaios clínicos, sendo excluídos os ensaios dos fármacos ainda não avaliados em estudos duplo-cegos. Foram selecionadas publicações nas línguas portuguesa e inglesa de artigos de revisão, ensaios clínicos e abstracts de encontros científicos e, para complementar a pesquisa bibliográfica, as referências dos artigos selecionados foram pesquisadas para buscar possíveis artigos não encontrados pela busca eletrônica ou artigos de valor histórico com data anterior a 1990.

\section{Potencialização de antidepressivos}

\section{Lítio}

Entre as estratégias de potencialização de antidepressivos, a do emprego de lítio foi a mais estudada. O mecanismo de ação desse fármaco ainda não foi adequadamente esclarecido, mas se demonstraram ações em várias etapas da neurotransmissão. Por exemplo, ações em sistemas de segundo mensageiro, na expressão gênica e na regulação da proteinoquinase $C$ (PCK). Foram demonstrados, ainda, efeitos neuroprotetores como regeneração de axônios e neurogênese hipocampal e proteção contra estímulos pró-apoptóticos (Bauer et al., 2003).

No caso da potencialização, mesmo que haja indefinição no que diz respeito ao modo de sua ocorrência, por efeitos de sinergismo ou por mecanismos antidepressivos (Heninger et al., 1983; Bauer et al., 2003), formulou-se a hipótese de que o lítio potencializaria a função serotonérgica pré-sináptica, facilitando a transmissão serotonérgica por aumento de turnover e liberação da serotonina em oposição à subsensibilização pós-sináptica induzida pelos antidepressivos nos receptores de serotonina (De Montigny et al., 1983; Bauer et al., 2003).

Nesta revisão foram encontrados 17 estudos duplo-cegos, randomizados, que incluíram, ao todo, 591 pessoas. Entre os estudos, 14 utilizaram tricíclicos, seis utilizaram inibidores seletivos da recaptação da serotonina (ISRS), dois utilizaram inibidores da monoamina oxidase (IMAO) e dois utilizaram outras classes de antidepressivos. Dos 14 trabalhos envolvendo tricíclicos, nove utilizaram apenas esses fármacos (De Montigny et al., 1983; Cournoyer et al., 1984; Kantor et al., 1986; Dinan e Barry 1989; Browne et al., 1990; Stein e Bernardt 1993; Joffe et al., 1993; Hoencamp et al., 1994; Nierenberg et al., 2003) e cinco envolveram dois ou mais antidepressivos (Heninger et al., 1983; Zusky et al., 1988; Schopf et al., 1989; Katona et al., 1995; Rybakowski et al., 1999). Os IMAO foram utilizados em apenas dois ensaios, provavelmente devido ao fato de em geral serem empregados em etapas mais tardias da resistência ao tratamento e, também, devido aos riscos inerentes aos mesmos (Tabela 1). Nesses estudos não houve comparação direta da potencialização com lítio entre diferentes classes de antidepressivos.

O lítio mostrou-se significativamente superior ao placebo em $41 \%$ (sete em 17) dos estudos considerados globalmente. Nos estudos apenas com tricíclicos, ele foi superior em $43 \%$ (três em sete); naqueles envolvendo tricíclicos e outras classes foi superior em $43 \%$ (três em sete) e nos estudos apenas com ISRS, em 14\% (um em sete).

Vários fatores devem ser considerados na análise da potencialização pelo lítio, como gravidade da depressão, cronicidade, número de episódios prévios, presença de co-morbidade, história de bipolaridade, tempo de depressão, grau de resistência, doses dos antidepressivos e níveis plasmáticos do lítio. Esses fatores foram analisados em poucos estudos e, freqüentemente, de forma isolada.

A relação entre a gravidade da depressão e a resposta à potencialização com o lítio foi analisada em seis estudos, mas os resultados foram controversos. Quatro associaram menor intensidade da depressão à boa resposta ao lítio (Zusky et al., 1988; Joffe et al., 1993a; Joffe et al., 1993b; Perlis et al., 2003) e dois associaram depressão mais grave à melhor resposta (De Montigny et al., 1983; Bschor et al., 2001). 
Tabela 1. Estudos com lítio duplo-cegos controlados

\begin{tabular}{|c|c|c|c|c|c|c|c|}
\hline Autor & $n$ & $\begin{array}{l}\text { Duração do } \\
\text { ensaio (dias) }\end{array}$ & $\begin{array}{c}\text { Antidepressivo } \\
\text { em uso (semanas) }\end{array}$ & $\begin{array}{c}\text { Dose de lítio (mg/dia)/ } \\
\text { nível sérico (mEq/l) }\end{array}$ & $\begin{array}{l}\text { Resposta ao } \\
\text { lítio (\%) }\end{array}$ & $\begin{array}{l}\text { Resposta ao } \\
\text { placebo (\%) }\end{array}$ & $p$ \\
\hline $\begin{array}{l}\text { De Montigny et al., } \\
1983\end{array}$ & $10 \mathrm{NE}$ & 2 & $\geq 150 \mathrm{mg} \mathrm{ADT}(\geq 3)$ & $900(0,4-1,2)$ & 100 & 20 & $<0,001$ \\
\hline Heninger et al., 1983 & $\begin{array}{l}14 \mathrm{UP} \\
1 \mathrm{BP}\end{array}$ & 12 & $\begin{array}{l}\geq 150 \mathrm{mg} \text { AMT equi- } \\
\text { valente ou } \geq 90 \mathrm{mg} \\
\text { mianserina }(\geq 3)\end{array}$ & $900-1.200(0,5-1,1)$ & 62 & 0 & $<0,014$ \\
\hline Curnoyer et al., 1984 & $12 \mathrm{NE}$ & 2 & ADT (3) & $900 \mathrm{ND}$ & Significativa & NS & ND \\
\hline Kantor et al., 1986 & 7 UP & 2 & $\begin{array}{c}\text { 100-250mg vários } \\
\text { ADTs }(\geq 3)\end{array}$ & $900 \mathrm{ND}$ & 25 & 0 & NS \\
\hline Zusky et al., 1988 & 16 UP & 14 & $\begin{array}{l}\text { 150mg IMP ou } \\
\text { equivalente ou } \\
\text { 60mg fenelzina/tra- } \\
\text { zodona }(\geq 4)\end{array}$ & $300-900(0,1-0,8)$ & 38 & 25 & NS \\
\hline Schopf et al., 1989 & $\begin{array}{l}18 \mathrm{UP} \\
9 \mathrm{BP}\end{array}$ & 14 & $\begin{array}{l}\text { Vários ADTs/fluvo- } \\
\text { xamina }(\geq 3)\end{array}$ & $600-800(0,4-1,2)$ & 50 & 0 & $<0,005$ \\
\hline Dinan e Barry, 1989 & $30 \mathrm{NE}$ & 21 & $\begin{array}{l}\text { 150mg AMT ou } \\
\text { equivalente (4) }\end{array}$ & $600-800(0,5-0,7)$ & 67 & 73 & NS \\
\hline Browne et al., 1990 & $\begin{array}{l}14 \mathrm{UP} \\
3 \mathrm{BP}\end{array}$ & 2 & $\begin{array}{c}\text { 150mg a } 300 \mathrm{mg} \\
\text { ADT ou } 150 \text { a } \\
\text { 200mg maprotilina } \\
(\geq 3)\end{array}$ & $900 \mathrm{ND}$ & 43 & 20 & NS \\
\hline Stein e Bernardt, 1993 & 34 UP & 63 & $\begin{array}{l}\text { 150mg AMT ou } \\
\text { equivalente }(\geq 3)\end{array}$ & $250-750(0,6-0,8)$ & $18-44$ & 22 & NS \\
\hline Joffe et al., 1993 & $50 \mathrm{NE}$ & 14 & $\begin{array}{c}2,5 \mathrm{mg} / \mathrm{kg} \text { tricíclico } \\
(5)\end{array}$ & $900-1.200(>0,55)$ & 53 & 19 & 0,038 \\
\hline Fava et al., 1994 & $41 \mathrm{NE}$ & 28 & $\begin{array}{l}\text { 20mg fluoxetina } \\
\qquad(\geq 8)\end{array}$ & $300-600(0,21)$ & 29 & - & 0,24 \\
\hline Hoencamp et al., 1997 & $51 \mathrm{NE}$ & 42 & Maprotilina (6) & $600-1.200(0,4-1,2)$ & 30 & - & 0,737 \\
\hline Katona et al., 1995 & $62 \mathrm{NE}$ & 42 & $\begin{array}{l}\text { 20mg fluoxetina } \\
\text { ou } 140 \mathrm{mg}-210 \mathrm{mg} \\
\text { lofepramina }(\geq 6)\end{array}$ & $\begin{array}{c}400+\text { ajuste até } 800 \\
(0,6-1)\end{array}$ & 52 & 25 & $<0,05$ \\
\hline Baumann et al., 1996 & $\begin{array}{l}23 \mathrm{DP}, \\
1 \mathrm{BP}\end{array}$ & 14 & $\begin{array}{c}\text { 40mg-60mg citalo- } \\
\text { pram }(\geq 4)\end{array}$ & $800+$ ajuste $(0,5-0,8)$ & 60 & 14 & $<0,05$ \\
\hline Rybakowski et al., 1999 & 59 UP/BP & 28 & $\begin{array}{l}\text { Tricíclico ou IMAO } \\
\text { ou ISRS em doses } \\
\text { terapêuticas }(\geq 6)\end{array}$ & $500-1.500(0,5-0,8)$ & 68 & - & NS \\
\hline Fava et al., 2002 & $101 \mathrm{NE}$ & 28 & 20mg fluoxetina (8) & $300-600(0,1-0,8)$ & 24 & - & NS \\
\hline Nierenberg et al., 2003 & $35 \mathrm{NE}$ & 42 & $\begin{array}{l}\geq 150 \mathrm{mg} \text { ADT ou } \\
\text { equivalente ou } \\
\text { outro em dose } \\
\text { adequada (6) }\end{array}$ & $-(0,6-0,9)$ & 11 & 18 & NS \\
\hline
\end{tabular}

ADT: antidepressivo tricíclico; NS: não-significativo; UP: depressão unipolar; BP: história de bipolaridade; NE: não-especificado; IMAO: inibidor da monoamina oxidase; ISRS: inibidor seletivo da recaptação da serotonina; AMT: amitriptilina; IMP: imipramina.

A melhor resposta ao emprego do lítio também foi associada a episódios agudos (Zusky et al., 1988; Rybakowski et al., 1999; Bschor et al., 2001), pacientes com menor número de episódios prévios (Rybakowski et al., 1999), ausência de transtorno de personalidade (Bschor et al., 2001), existência de histórico pessoal (Heninger et al., 1983) ou familiar (Zusky et al., 1988) de bipolaridade, histórico de início precoce da depressão e estado civil casado (Perlis et al., 2003). Com relação ao grau de refratariedade, foi sugerido que o emprego do lítio como potencializador de antidepressivos em pacientes com depressão resistente a múltiplos tratamentos teria eficácia limitada (Nierenberg et al., 2003). No entanto, nos sete estudos revistos que demonstraram melhora significativa com o uso do lítio esse fator não foi estudado (Tabela 1). Verificou-se que todos os ensaios utilizaram como critério de inclusão a resistência a pelo menos um antidepressivo, mas nenhum deles abordou o nível de resistência na análise dos dados.

As doses dos antidepressivos variaram dentro de variações estabelecidas como terapêuticas, e não houve relação entre dose do antidepressivo e potencialização pelo lítio. No caso dos tricíclicos, seus níveis plasmáticos não interferiram na intensidade da resposta à potencialização pelo lítio (Browne et al., 1990; Stein e Bernardt, 1993; Fava et al., 2002). Também observou-se que o lítio não alterou os níveis plasmáticos dos antidepressivos (De Montigny et al., 1983; Browne et al. 1990; Joffe et al., 1993; Baumann et al., 1996) (Tabela 1). No que diz respeito aos níveis plasmáticos do lítio, os estudos de De Montigny et al. (1983), Heninger et al. (1983), Cournoyer et 
al. (1984), Shopf (1989), Rybakowisky et al. (1999), Bauer et al. (2003) e Fava et al. (2002) não verificaram relação entre resposta e níveis séricos terapêuticos, diferentemente do que foi evidenciado nos ensaios clínicos de Stein e Bernardt (1993) e Fava et al. (1994) e sugerido por Zusky et al. (1988). Apesar da controvérsia, recomenda-se a utilização do lítio em níveis terapêuticos (Rouillon e Gorwood, 1998).

Quanto ao tempo de latência, embora haja relato de melhora significativa em apenas 48h após adição de lítio (De Montigny et al., 1983), observou-se que a maioria dos pacientes apresentou melhora após a primeira (Heninger et al., 1983; Stein e Bernardt, 1993; Joffe et al., 1993; Baumann et al., 1996), a segunda (Nierenberg et al., 2003) ou a sexta semana de potencialização (Katona et al., 1995). Portanto, tendo em vista essas variações no tempo de resposta, sugeriu-se o emprego do lítio por, no mínimo, três (Rouillon e Gorwood, 1998) a seis semanas (Katona et al., 1995). A duração do tratamento de manutenção com o lítio ainda não foi devidamente estudada. Boa resposta inicial a esse fármaco foi associada a melhor resultado terapêutico em longo prazo (Nierenberg et al., 1990). Recomendase 0 tratamento de manutenção pelo período mínimo de seis a 12 meses (Bschor et al., 2001; Bauer et al., 2003).

Tremor, náusea, poliúria e ganho de peso são eventos adversos no emprego do lítio e podem ocorrer durante o tratamento (Zusky et al., 1988; Rouillon e Gorwood, 1998). De uma maneira geral, não houve piora dos eventos adversos apresentados pelos pacientes quando ele foi acrescido ao tratamento antidepressivo (Baumann et al., 1996). Essa associação foi bem tolerada, mas recomendou-se o controle da função tireoidiana, devido ao risco de hipotireoidismo, e o controle dos níveis plasmáticos do lítio pelo risco de intoxicação (Sussman e Joffe, 1998).

Algumas limitações podem ser apontadas nos estudos de potencialização com o lítio. A maioria dos ensaios utilizou amostras pequenas (De Montigny et al., 1983; Heninger et al., 1983; Cournoyer et al., 1984; Kantor et al., 1986; Zusky et al., 1988; Schopf et al., 1989; Kramlinger e Post, 1989; Dinan e Barry, 1989; Browne et al., 1990, Stein e Bernardt, 1993; Baumann et al., 1996; Nierenberg et al., 2003), alguns incluíram pacientes que não fizeram uso do antidepressivo pelo período de tempo mínimo preconizado (Heninger et al., 1983; Montigny et al., 1981; De Montigny et al., 1983; Kantor et al., 1986), outros não foram controlados com placebo (Hoencamp et al., 1994; Fava et al., 1994 e 2002) e vários incluíram e analisaram em conjunto pacientes com história de episódio(s) de mania ou hipomania (Heninger et al., 1983; Dinan e Barry, 1989; Schopf et al., 1989; Hoencamp et al., 1994; Katona et al., 1995; Baumann et al., 1996; Stein e Bernardt, 1993).

Como a maioria dos estudos incluiu pequeno número de pacientes, foi realizada uma metanálise sobre o uso do lítio na potencialização de antidepressivos (Bauer e Dopfmer, 1999). Esse estudo incluiu dez ensaios duplo-cegos, envolvendo a potencialização de tricíclicos, IMAOs e ISRSs. Encontrou-se uma média de resposta ao lítio de $52 \%$, com odds ratio de 3,31 em relação ao placebo. Concluiuse que o lítio é eficaz na potencialização de antidepressivos.

Também foram realizadas duas revisões sistemáticas sobre a potencialização de antidepressivos com o lítio (Stimpson et al., 2002; Bauer et al., 2003). Stimpson et al. (2002) adotaram um crivo de seleção de artigos rigoroso e incluíram apenas dois ensaios envolvendo tricíclicos e IMAOs (Zusky et al., 1988; Joffe et al., 1993). Relataram que, embora encontrado um benefício estatisticamente significativo para o grupo do lítio, o intervalo de confiança (IC) foi muito amplo: de $2 \%$ a $49 \%$. Consideraram que as evidências do uso do lítio na potencialização de antidepressivos foram fracas. Bauer et al. (2003), incluindo número maior de artigos em sua revisão, mesmo após apontarem as limitações metodológicas dos estudos, consideraram suficientes as evidências da eficácia do lítio e recomendaram seu uso como estratégia de primeira linha na potencialização de tricíclicos, de IMAOs e de ISRSs.

Em síntese, as evidências sugerem que o lítio é eficaz na potencialização de antidepressivos. Embora alguns estudos tenham avaliado os preditores de resposta terapêutica, os dados sobre a gravidade da depressão foram controversos e os sobre cronicidade, número de episódios prévios, presença de co-morbidades, história de bipolaridade, tempo de depressão e grau de resistência foram escassos. Algumas outras questões também permanecem sem resposta: mecanismo de ação do lítio, o tempo necessário para início da resposta, níveis plasmáticos eficazes do lítio, duração do tratamento, eficácia em determinados subtipos de depressão, quando optar por seu uso na DRT e a eficácia na potencialização de outros antidepressivos.

\section{Hormônio tireoidiano}

A utilização do hormônio tireoidiano para a potencialização de antidepressivos no tratamento da DRT foi a segunda estratégia mais estudada. Os mecanismos de ação sugeridos foram a melhora na neurotransmissão noradrenérgica e os efeitos no eixo tireoidiano. Uma das ações no eixo tireoidiano seria a de correção da hipofunção tireoidiana em pacientes com hipotireoidismo subclínico. Outra seria a de que o emprego de L-triodotironina $\left(\mathrm{T}_{3}\right)$ levaria a uma redução de tiroxina $\left(\mathrm{T}_{4}\right)$. Baixos níveis de $\mathrm{T}_{4}$ plasmático acarretam baixos níveis de $\mathrm{T}_{3}$ cerebral, o que estaria relacionado a efeitos antidepressivos (Joffe e Singer, 1990; Joffe, 1998).

Foram avaliados seis estudos duplo-cegos que incluíram 243 pacientes (Tabela 2).

O uso do hormônio tireoidiano foi eficaz na potencialização de tricíclicos em $50 \%$ dos estudos revistos, sendo que em um deles (Joffe et al., 1993) a $\mathrm{T}_{3}$ foi equivalente ao lítio e superior ao placebo ( $p$ $=0,02)$. $A T_{3}$ foi mais freqüentemente utilizada que $\mathrm{T}_{4}$ nos estudos de potencialização. Um ensaio verificou superioridade da $T_{3}$ sobre a $\mathrm{T}_{4}$ (Joffe e Singer, 1990). Levantaram-se hipóteses para explicar esse resultado. A primeira relacionou-se com a menor meia-vida da $T_{3}$ (12h) em relação à T4 (uma semana). Como o ensaio durou apenas duas semanas, a T4 levaria um tempo maior até atingir o estado de equilíbrio necessário para se obterem efeitos potencializadores de antidepressivos. A segunda hipótese foi a de que os tricíclicos interfeririam na conversão de $\mathrm{T}_{4} \mathrm{em} \mathrm{T}_{3}$. Essa hipótese não foi comprovada em ensaios. A terceira foi a de que $T_{3}$ e $T_{4}$ teriam diferentes mecanismos de ação, porém desconhecidos. Existe, ainda, a hipótese de que $T_{3}$ e $T_{4}$ atuariam de maneira antagônica sobre os níveis de hormônio tireoidiano no cérebro, levando a diferentes efeitos no humor - a T3 exógena levaria a uma redução da $\mathrm{T}_{4}$ plasmática, que está diretamente relacionada à determinação dos níveis de $T_{3}$ no cérebro. Níveis menores de $T_{3}$ no cérebro estiveram relacionados a efeitos antidepressivos. Assim a $\mathrm{T}_{3}$ administrada por via exógena estaria relacionada a efeitos antidepressivos e $a T_{4}$, não (Joffe e Singer, 1990).

Com exceção da gravidade da depressão, outros fatores preditores de resposta ao emprego de $T_{3}$ na potencialização de 
Tabela 2. Estudos duplo-cegos com hormônio tireoidiano

\begin{tabular}{|c|c|c|c|c|c|c|c|}
\hline Autor & $n$ & $\begin{array}{l}\text { Duração do } \\
\text { ensaio (semanas) }\end{array}$ & $\begin{array}{c}\text { Antidepressivo } \\
\text { em uso } \\
\text { (semanas) }\end{array}$ & $\begin{array}{l}\text { Dose de } \\
\text { hormônio } \\
\text { ( } \mu \mathrm{g} / \mathrm{dia} \text { ) }\end{array}$ & Resposta à $\mathrm{T}_{3}(\%)$ & $\begin{array}{l}\text { Resposta ao } \\
\text { controle (\%) }\end{array}$ & $p$ \\
\hline $\begin{array}{l}\text { Steiner et al., } \\
\quad 1978\end{array}$ & 8 UP, 4 BP & 5 & 150mg IMP (NE) & 25 & $3 / 4(75)$ & $\begin{array}{c}\text { 3/4 (75) placebo, } \\
\text { 3/4 (75) ECT }\end{array}$ & NS \\
\hline $\begin{array}{l}\text { Goodwin et al., } \\
1982\end{array}$ & 4 UP, 8 BP & 3 & $\begin{array}{l}\text { 150-300mg de } \\
\text { IMP ou AMT (5) }\end{array}$ & $25-50$ & $9 / 12(75)$ & 0/6 (0) placebo & $<0,02$ \\
\hline Gitlin et al.,1987 & 16 UP & 4 & $\begin{array}{l}\text { IMP até } 300 \mathrm{mg} \\
\text { (NE) }\end{array}$ & 25 & $0 / 7(0)$ & 4/9 (44) placebo & 0,12 \\
\hline $\begin{array}{c}\text { Joffe e Singer, } \\
1990\end{array}$ & 40 UP & 3 & ADT (4) & $\begin{array}{l}37,5 \mathrm{~T}_{3} \\
150 \mathrm{~T}_{4}\end{array}$ & $\begin{array}{l}9 / 17(53) T_{3} \\
4 / 21(19) T_{4}\end{array}$ & - & 0,03 \\
\hline Joffe et al., 1993 & 50 UP & 2 & ADT (5) & 37,5 & 10/17 (59) & $\begin{array}{c}\text { 3/16 (19) } \\
\text { placebo } \\
\text { 9/17 (53) lítio }\end{array}$ & 0,02 \\
\hline Fliers, 2003 & 113 (NR) & 8 & $\begin{array}{l}\text { 30mg paroxetina } \\
\text { (NE) }\end{array}$ & $25-50$ & $(46)$ & (46) & NS \\
\hline Total & 243 & & & & & & \\
\hline
\end{tabular}

NS: não-significativo; UP: depressão unipolar; BP: história de bipolaridade; NE: não-especificado; $\mathrm{T}_{3}$ : triiodotironima; $\mathrm{T}_{4}$ : tiroxina; $\mathrm{AMT}$ : amitriptilina; ECT: eletroconvulsoterapia.

antidepressivos não foram estudados. Ainda assim, a relação entre gravidade da depressão e potencialização com $T_{3}$ até o momento não foi esclarecida. Tal aspecto foi abordado em apenas um estudo sobre preditores de resposta ao hormônio tireoidiano, que não encontrou diferenças entre respondedores e não-respondedores (Joffe et al., 1993). Em relação à possibilidade de interação medicamentosa, os níveis séricos dos antidepressivos tricíclicos não foram alterados pelo uso do hormônio tireoidiano (Goodwin et al., 1982; Joffe et al., 1993). A dose do hormônio utilizada foi entre 25 e $50 \mu \mathrm{g} / \mathrm{dia}$ e não houve evidência de superioridade entre elas (Goodwin et al., 1982). No entanto, foi preconizada a dose de $25 \mu \mathrm{g} / \mathrm{dia}$, que seria mais segura quanto aos riscos de alterações na função tireoidiana (Joffe, 1998).

A resposta à potencialização de antidepressivos com 0 hormônio tireoidiano ocorreu até a terceira semana (Sussman e Joffe, 1998). Seu tempo de uso ainda não foi devidamente estudado (Aronson et al., 1996). Entretanto recomenda-se manter o hormônio tireoidiano enquanto o paciente estiver em uso de antidepressivos (Joffe, 1998).

No que diz respeito à segurança, o hormônio tireoidiano foi bem tolerado. Os efeitos colaterais mais comuns foram nervosismo e insônia (Fava, 2000). Dado o risco de hipertireoidismo, recomendou-se a monitoração da função tireoidiana antes e após o uso do hormônio (Joffe, 1998).

Como os estudos com antidepressivos tricíclicos incluíram número pequeno de pacientes nos grupos de uso hormônio tireoidiano (Goodwin et al., 1982; Joffe e Singer, 1990; Joffe et al., 1993), foi realizada uma metanálise (Aronson et al., 1996), que demonstrou a superioridade da $\mathrm{T}_{3}$ em relação ao placebo $(p=0,02)$. Apenas um estudo avaliou a potencialização de ISRS, no caso a paroxetina, apresentando resultado negativo (Fliers, 2003). Assim, ficou evidenciada a eficácia do hormônio tireoidiano apenas na potencialização de tricíclicos. As maiores limitações observadas nos estudos duplo-cegos sobre a eficácia do uso do hormônio tireoidiano como potencializador de antidepressivos foram o número reduzido de ensaios, a utilização de amostras pequenas (Steiner et al., 1978; Goodwin et al., 1982; Gitlin et al., 1987; Joffe et al., 1993), a inclusão de pacientes bipolares (Steiner et al., 1978; Goodwin et al., 1982) e a curta duração do ensaio (Joffe et al., 1993).

Concluindo, o hormônio tireoidiano foi considerado eficaz na potencialização de antidepressivos tricíclicos. Apesar de bem tolerado, seguro e eficaz, seu tempo de uso, seus preditores de resposta terapêutica e a sua associação a antidepressivos de nova geração ainda não foram adequadamente estudados.

\section{Buspirona}

A buspirona é uma droga agonista parcial de receptores serotonérgicos, com fracas propriedades ansiolíticas. Em relatos de casos (Bakish, 1991) e em estudos abertos não-controlados (Jacobsen, 1991; Joffe e Schuller, 1993; Fisher et al., 1998; Dimitriou e Dimitriou, 1998) observou-se que a buspirona aumentou os efeitos antidepressivos dos ISRSs. Além desses relatos de casos e estudos abertos, foram realizados dois estudos duplo-cegos, randomizados e controlados com placebo, e um comparativo, não-controlado com placebo, avaliando a buspirona e a bupropiona como potencializadores de ISRS. O primeiro estudo duplo-cego, randomizado e controlado com placebo (Landén et al., 1998) durou quatro semanas e envolveu 117 pacientes de doze centros da Suécia e um da Noruega, incluindo 102 pacientes unipolares e bipolares, resistentes a quatro semanas de $30 \mathrm{mg}$ de paroxetina ou $40 \mathrm{mg}$ de citalopram. Cinqüenta e um por cento dos pacientes que utilizaram buspirona e $47 \%$ dos pacientes do grupo placebo melhoraram com o tratamento. Não houve diferença significativa entre esses grupos. Entretanto, em follow-up aberto de duas semanas, 69,5\% dos pacientes responderam à associação ISRS + buspirona. O segundo estudo duplo-cego, randomizado e controlado com placebo, realizado em três centros na Finlândia (Appelberg et al., 2001), avaliou durante seis semanas 102 pacientes deprimidos unipolares que eram resistentes a seis semanas de fluoxetina $30 \mathrm{mg}$ ou citalopram $40 \mathrm{mg}$. Na primeira semana o grupo em uso da buspirona obteve melhora significativa em relação ao placebo, diferença que não foi mantida ao longo das seis semanas do estudo. Assim, embora não tenha sido encontrada diferença significativa com relação ao placebo, foi observada aceleração na resposta ao tratamento em pacientes resistentes à fluoxetina e ao 
citalopram. O estudo duplo-cego, randomizado e não-controlado com placebo sobre o uso da buspirona e da bupropiona como potencializador de ISRSs avaliou 286 pacientes resistentes a 11,9 semanas de citalopram numa dose média de $55 \mathrm{mg}$ e não encontrou redução significativa dos sintomas depressivos na avaliação pela Escala de Depressão de Hamilton (HDRS) (1960).

A relação entre gravidade da depressão e eficácia do emprego da buspirona na potencialização de antidepressivos foi avaliada em apenas um estudo. Nesse trabalho os autores observaram que os pacientes com pontuação inicial mais elevada na escala de depressão de Montgomery e Asberg (MADRS) (1979) tenderam a uma maior redução da pontuação final nessa mesma escala. Isso indicou que a buspirona poderia ser mais útil como opção de tratamento de pacientes graves, resistentes a ISRSs (Appelberg et al., 2001). Não foram estudados outros preditores de resposta terapêutica, como cronicidade da depressão, número de episódios prévios, presença de co-morbidades psiquiátricas, história de bipolaridade, tempo de depressão, grau de resistência, tempo de latência para a resposta e tempo de tratamento da buspirona na potencialização de antidepressivos.

Landén et al. (1998) observaram aumento nos níveis plasmáticos de citalopram e paroxetina tanto nos pacientes em uso da buspirona quanto naqueles em uso do placebo. $O$ aumento foi atribuído à possibilidade de maior adesão ao tratamento antidepressivo pelos pacientes incluídos no estudo ou, no caso da associação buspirona/antidepressivo, à interação farmacocinética.

Nos estudos duplo-cegos, as doses de buspirona variaram de 20 a $60 \mathrm{mg}$. Os efeitos colaterais mais comuns foram sedação, cefaléia e sudorese, e nenhum foi considerado grave. Foram relatados dois casos de síndrome serotonérgica. Num deles o paciente estava utilizando buspirona e citalopram (Spigset e Adielsson, 1997) e no outro, buspirona e fluvoxamina (Baetz e Malcolm, 1995). O mesmo paciente em uso da associação buspirona/citalopram desenvolveu hiponatremia (Spigset e Adielsson, 1997). Houve relato de um paciente que entrou em mania, achado que corroborou a hipótese de ação antidepressiva da buspirona (Sussman e Jaffe, 1998). Apesar dos eventos adversos, a associação de buspirona a antidepressivos ISRS foi bem tolerada (Jacobsen, 1991; Fisher et al., 1998; Landén et al., 1998; Appelberg et al., 2001). No entanto, recomenda-se cautela na associação de buspirona a antidepressivos que inibem a enzima 3A3/4 do sistema citocromo P450, como a nefazodona e a fluvoxamina (Rocha e Hara, 1998).

Os três estudos duplo-cegos controlados existentes não constataram sua eficácia na potencialização de antidepressivos. Pode-se concluir que a buspirona foi pouco estudada e que os estudos existentes não sugerem que ela possa ter papel mais relevante em DRT.

\section{Pindolol}

$O$ pindolol é um agente betabloqueador, agonista parcial e antagonista de receptores 5-HT1A somatodendríticos. Esses receptores servem como inibidores de auto-receptores e reduzem a síntese e a liberação de serotonina no terminal nervoso. A administração aguda de ISRSs aumenta a concentração de serotonina na fenda sináptica e, ao mesmo tempo, ativa auto-receptores 5-HT1A inibitórios ( feedback negativo). O pindolol tem sido utilizado com o objetivo de potencializar antidepressivos com ação primária em receptores serotonérgicos. O mecanismo de ação proposto seria o de bloquear o feedback negativo e permitir maior liberação de serotonina.

Em ensaios abertos, observou-se que o pindolol induziu melhora dramática em pacientes resistentes a ISRSs e IMAOs (Artigas et al., 1994; Blier e Bergeron, 1998). Em estudos controlados, entretanto, a eficácia do pindolol não foi comprovada. Os resultados foram controversos (Tabela 3).

Os estudos duplo-cegos totalizaram 205 pacientes. Um dos ensaios avaliou o emprego do pindolol na potencialização da trazodona (Maes et al., 1996); três na potencialização de ISRS (Maes et al., 1999; Sokolski et al., 2004; Perry et al., 2004) e dois na potencialização de ISRS e tricíclicos (Moreno et al., 1997; Perez et al., 1999; Perry et al., 2004). Em três estudos (Maes et al., 1996; Maes et al., 1999; Sokolski et al., 2004) foi encontrada diferença significativa entre associação de pindolol a antidepressivos versus placebo, e em três (Moreno et al., 1997; Perez et al., 1999; Perry et al., 2004) não foi evidenciada tal diferença. Como não existem estudos comparando diretamente a eficácia do pindolol em potencializar diferentes classes de antidepressivos, não é possível afirmar se há diferença na potencialização das diversas classes (Tabela 3).

Os preditores de resposta não foram avaliados em estudos específicos. Apenas um ensaio sobre a eficácia do pindolol na potencialização de antidepressivos abordou tempo de depressão, número de episódios prévios, duração do episódio depressivo atual e fatores sociodemográficos. Porém, o estudo não encontrou influência dos mesmos na resposta à potencialização de antidepressivos com o pindolol (Maes et al., 1996). Não foram estudados cronicidade, presença de co-morbidade e grau de refratariedade.

Adose de pindolol utilizada nos estudos foi $2,5 \mathrm{mg}$, três vezes ao dia. Em alguns dos ensaios duplo-cegos observou-se celeração na resposta aos ISRSs na primeira e na segunda semana (Moreno et al., 1997; Perez et al., 1999; Maes et al., 1999). A duração do tratamento não foi estudada.

Os eventos adversos mais comumente observados foram náusea, tonteira, sedação, hipotensão e bradicardia. Esses efeitos não foram graves e a potencialização de antidepressivos com 0 pindolol foi bem tolerada. Entretanto recomenda-se cautela na prescrição desse fármaco em pacientes asmáticos, alérgicos graves e portadores de problemas na condução cardíaca. O seu uso em diabéticos é uma contra-indicação relativa, devido à possibilidade de mascaramento de sintomas de hipoglicemia (Blier e Bergeron, 1998).

As principais falhas encontradas nos estudos duplo-cegos sobre o uso do pindolol na potencialização de antidepressivos foram 0 uso de amostras pequenas (Maes et al., 1996; Moreno et al., 1997; Maes et al., 1999; Sokolski et al., 2004) e a não-explicitação sobre inclusão ou não de pacientes com história de bipolaridade (Maes et al., 1996; Maes et al., 1999; Sokolski et al., 2004).

Foi realizada metanálise sobre o emprego do pindolol na potencialização de antidepressivos (Stimpson et al., 2002), incluindo três estudos duplo-cegos (Maes et al., 1996; Moreno et al., 1997; Perez et al., 1999). Nesse trabalho o pindolol não se mostrou eficaz.

Pode-se concluir que os estudos duplo-cegos sobre a potencialização com o pindolol são escassos e os resultados são controversos, impedindo conclusões definitivas sobre sua eficácia.

\section{Carbamazepina}

O mecanismo de ação da carbamazepina foi relacionado com redução do influxo de sódio, facilitação do efluxo do potássio, 
Tabela 3. Estudos duplo-cegos com pindolol

\begin{tabular}{|c|c|c|c|c|c|c|c|}
\hline Autor & $n$ & $\begin{array}{l}\text { Duração do } \\
\text { ensaio (dias) }\end{array}$ & $\begin{array}{c}\text { Antidepressivo em uso } \\
\text { (semanas) }\end{array}$ & $\begin{array}{l}\text { Dose pindolol } \\
\text { (mg/dia) }\end{array}$ & Resposta (\%) & $\begin{array}{l}\text { Resposta } \\
\text { placebo (\%) }\end{array}$ & $p$ \\
\hline Maes et al., 1996 & $33 \mathrm{NE}$ & 28 & 100mg trazodona (NE) & 7,5 & $8 / 11(73)$ & $2 / 10(20)$ & 0,02 \\
\hline Moreno et al., 1997 & 10 UP & 14 & $\begin{array}{l}20-40 \mathrm{mg} \text { fluoxetina, } 300 \mathrm{mg} \\
\text { desipramina, } 450 \mathrm{mg} \text { bupro- } \\
\text { piona }(\geq 8)\end{array}$ & 7,5 & $0 / 10(0)$ & $2 / 10(20)$ & NS \\
\hline Perez et al., 1999 & 80 UP & 10 & $\begin{array}{l}\text { 150mg clomipramina, } 40 \mathrm{mg} \\
\text { fluoxetina, } 40 \mathrm{mg} \text { paroxetina, } \\
200 \mathrm{mg} \text { fluvoxamina }(\geq 2)\end{array}$ & 7,5 & $5 / 40(13)$ & $5 / 40(13)$ & NS \\
\hline Maes et al., 1999 & $31 \mathrm{NE}$ & 35 & 20mg fluoxetina (NE) & 7,5 & $6 / 10(60)$ & - & 0,007 \\
\hline $\begin{array}{l}\text { Sokolski et al., } \\
2004\end{array}$ & $9 \mathrm{NE}$ & 28 & $40 \mathrm{mg}$ paroxetina $(\geq 8)$ & 7,5 & $4 / 4(100)$ & $0 / 5(0)$ & 0,001 \\
\hline Perry et al., 2004 & 42 UP & 42 & $\begin{array}{l}20 \mathrm{mg} \text { fluoxetina, } 20 \mathrm{mg} \text { paro- } \\
\text { xetina, } 50 \mathrm{mg} \text { sertralina (6) }\end{array}$ & 7,5 & $4 / 21(19)$ & $4 / 17(24)$ & NS \\
\hline Total & 205 & & & & & & \\
\hline
\end{tabular}

NS: não-significativo; UP: depressão unipolar; NE: não-especificado.

inibição dos canais de cálcio e subseqüente redução da liberação de aminoácidos excitatórios. Após sua administração crônica, ela demonstrou efeitos noradrenérgicos, dopaminérgicos e moduladores do sistema GABAérgico, que aumentaram os níveis de GABA no cérebro e no liquor. No entanto, não se sabe como a carbamazepina atuaria nos transtornos do humor (Post et al., 1998).

Em DRT existe um estudo duplo-cego, comparativo, nãocontrolado com placebo sobre o uso da carbamazepina e do lítio na potencialização de antidepressivos (Rybakowski et al., 1999). Nesse ensaio foram avaliados durante quatro semanas 59 pacientes deprimidos uni e bipolares (28 pacientes utilizaram a carbamazepina e 31 lítio). Houve resposta terapêutica em $50 \%$ dos pacientes em uso da carbamazepina, e sua eficácia foi equiparada à do lítio.

Quanto à gravidade da doença, observou-se que os respondedores à carbamazepina apresentaram um grau de depressão mais brando em comparação aos não-respondedores. Não foram estudados outros preditores de resposta terapêutica como cronicidade, número de episódios depressivos prévios, duração do episódio atual, presença de co-morbidade e grau de refratariedade. A dose de carbamazepina utilizada variou de 400 a $800 \mathrm{mg}$. Houve boa tolerabilidade, mas deve-se estar atento aos riscos de interações medicamentosas (Dietrich e Emrich, 1998).

Concluindo, a carbamazepina foi muito pouco estudada em DRT, de modo que sua eficácia e sua segurança na potencialização de antidepressivos ainda não foram esclarecidas.

\section{Lamotrigina}

O mecanismo de ação da lamotrigina ainda não foi totalmente elucidado. Propôs-se que ela estabiliza membranas neuronais e, conseqüentemente, modula a liberação pré-sináptica de aminoácidos excitatórios, particularmente glutamato. Estudos com lamotrigina demonstraram sua eficácia nos transtornos bipolares, e foram observados particularmente efeitos antidepressivos (Calabrese et al., 1998; Calabrese et al., 1999; Fava, 2000, Bowden et al., 2003). Na potencialização de antidepressivos esse anticonvulsivante foi estudado em um relato de caso (Maltese, 1999), dois ensaios retrospectivos (Barbee e Jamhour, 2002; Rocha e Hara, 2003) e um estudo duplo-cego de potencialização de antidepressivos em pacientes com DRT unipolar (Barbosa et al., 2003). Nesse estudo o emprego da lamotrigina na potencialização de antidepressivos foi eficaz em $52 \%$ dos pacientes. Não foi evidenciada eficácia da lamotrigina na potencialização da fluoxetina quando se utilizou a avaliação pelo critério principal (escalas HDRS e MADRS). Entretanto a lamotrigina foi estatisticamente superior ao placebo em critérios secundários de eficácia (Impressão Clinica Global [CGI]). Segundo os autores, a existência de bipolaridade não influenciou os resultados do tratamento, e a cronicidade da depressão não foi avaliada (Barbosa et al., 2003).

Embora tenha sido demonstrada superioridade terapêutica da dose de $200 \mathrm{mg}$ da lamotrigina (Calabrese et al., 1999), a dose empregada nesse ensaio foi $100 \mathrm{mg}$. Houve relato de dois casos de leucopenia (Normann et al., 2003) e um de hipomania (Barbosa et al., 2003). Recomenda-se atenção quanto aos riscos de síndrome de Stevens-Johnson (pode ocorrer em $0,1 \%$ a $0,3 \%$ dos casos) e interação medicamentosa. O valproato reduz em $50 \%$ o clearance da lamotrigina, aumentando sua meia-vida de eliminação. Há relato de dois casos de interação da lamotrigina com sertralina em que ocorreu elevação dos níveis séricos da lamotrigina e neurotoxicidade (Jefferson, 2005). Recomenda-se, ainda, que ao iniciar o uso da lamotrigina seja feito 0 aumento gradual da dose, para reduzir a probabilidade de rash cutâneo (Jefferson, 2005). Apesar dos eventos mencionados, a lamotrigina foi bem tolerada pela maioria dos pacientes (Barbosa et al., 2003). Algumas limitações poderiam explicar o resultado negativo desse estudo: amostra pequena (23 pacientes), heterogênea (unipolares e bipolares II) e dose máxima de lamotrigina de $100 \mathrm{mg}$.

Assim, conclui-se que a utilidade da lamotrigina na potencialização de antidepressivos em pacientes com DRT ainda não foi adequadamente estudada. Há evidência menor de eficácia quando considerado critério secundário de avaliação.

\section{Olanzapina e outros antipsicóticos atípicos}

O tratamento da DRT com associação de antipsicóticos típicos já foi estudado. Houve achados promissores, mas de uso limitado devido ao risco de discinesia tardia (Nelson, 2003). Com a introdução dos antipsicóticos atípicos, retornou o interesse 
em avaliar o uso dos antipsicóticos no tratamento de pacientes com DRT. A primeira publicação, de Ostroff e Nelson (1999), que sugeriu uma possível utilidade da risperidona como agente potencializador de antidepressivos, baseou-se na idéia de que a adição de um antagonista de receptores 5 -HT2A poderia aumentar 0 efeito de ISRS. Sendo o receptor 5-HT2A excitatório, que age em oposição aos receptores 5-HT1A pós-sinápticos, o seu antagonismo poderia facilitar a ação da serotonina nos receptores 5-HT1A.

Existem relatos de casos sobre o emprego da risperidona (Tani et al., 2004; Stoll e Haura, 2000; Ostroff e Nelson, 1999) como potencializador; quatro estudos abertos avaliaram a ziprasidona (Papakostas et al., 2004; Papakostas et al., 2005 [abstract]; Simon e Nemeroff, 2005 [abstract]); um estudo aberto avaliou a olanzapina (Corya et al., 2003). Realizaram-se cinco estudos duplo-cegos, com um total de 1.530 pacientes, em relação à olanzapina como agente de potencialização de antidepressivos (Tabela 4).

Em dois estudos o emprego da olanzapina associada à fluoxetina foi superior ao emprego da fluoxetina ou da olanzapina em monoterapia (Shelton et al., 2001; Corya et al., 2006). Outros não observaram essa diferença (Dubé et al., 2002a; Dubé et al., 2002b; Shelton et al., 2005). Observou-se, porém, que os pacientes em uso da combinação fluoxetina/olanzapina obtiveram maior rapidez de resposta nas primeiras semanas de tratamento (Shelton et al., 2001; Shelton et al., 2005).

Fatores como gravidade da depressão, cronicidade, tratamento prévio, presença de transtornos psiquiátricos concomitantes, história de bipolaridade e grau de resistência não foram analisados nos estudos duplo-cegos.
A dose de olanzapina utilizada variou de 5 a $20 \mathrm{mg}$. Os eventos adversos mais observados foram aumento do apetite, ganho de peso, sonolência, astenia, cefaléia, tremores, ataxia, nervosismo, insônia e náusea. Com relação ao aumento de apetite e ganho de peso, a combinação olanzapina/fluoxetina foi equiparada à monoterapia com olanzapina e superior às com fluoxetina e nortriptilina (Shelton, 2001; Shelton et al., 2005). Foi relatado um caso de síndrome serotonérgica após associação de fluoxetina e olanzapina (Chopra e Schweitzer, 2003). Em geral, a combinação de olanzapina com ISRSs foi bem tolerada. Na prescrição de olanzapina são recomendados cuidados para prevenir o ganho de peso e o desenvolvimento de síndrome metabólica (Nasrallah e Newcomer, 2004).

As maiores limitações encontradas para o uso de antipsicóticos na potencialização de antidepressivos foram o pequeno número de ensaios, a existência de ensaios duplo-cegos apenas com a olanzapina e a não-informação sobre a inclusão ou não de pacientes bipolares (Dubé et al., 2002a, Dubé et al., 2002b).

Dois estudos foram favoráveis ao uso da olanzapina, entretanto três grandes ensaios não evidenciaram sua superioridade em relação ao placebo. Assim, a eficácia da olanzapina como potencializador de fluoxetina em DRT não está estabelecida. Quanto aos outros antipsicóticos atípicos, ensaios duplo-cegos são necessários para que se chegue a alguma conclusão.

\section{Discussão}

Os dois potencializadores de antidepressivos mais estudados - o lítio e, em menor escala, o hormônio tireoidiano - tiveram sua eficácia estabelecida. Aolanzapina foi razoavelmente estudada, mas não foi comprovada a sua eficácia na potencialização de antidepres-

Tabela 4. Estudos duplo-cegos com olanzapina

\begin{tabular}{|c|c|c|c|c|c|c|c|}
\hline Autor & $n$ & $\begin{array}{l}\text { Antidepressivo em } \\
\text { potencialização } \\
\text { (semanas) }\end{array}$ & $\begin{array}{l}\text { Olanzapina } \\
\text { (mg/dia) }\end{array}$ & $\begin{array}{l}\text { Duração } \\
\text { (semanas) }\end{array}$ & $\begin{array}{l}\text { Resposta antidepressivol } \\
\text { olanzapina (\%) }\end{array}$ & Controle (\%) & $\begin{array}{c}p \\
\text { potencialização }\end{array}$ \\
\hline Shelton et al., 2001 & 28 UP & 52mg fluoxetina (6) & $5-20$ & 8 & $\begin{array}{l}\text { Fluoxetina/olanzapina 6/10 } \\
\qquad(60)\end{array}$ & $\begin{array}{c}\text { Fluoxetina/ } \\
\text { placebo (10), } \\
\text { olanzapina/pla- } \\
\text { cebo }(0)\end{array}$ & $<0,05$ \\
\hline $\begin{array}{l}\text { Dubé et al., 2002b } \\
\text { (Shelton, 2004) }\end{array}$ & $499 \mathrm{NE}$ & Fluoxetina (NE) & NE & 8 & Fluoxetina/olanzapina (NR) & $\begin{array}{l}\text { Fluoxetina, } \\
\text { olanzapina, } \\
\text { nortriptilina }\end{array}$ & NS \\
\hline $\begin{array}{l}\text { Dubé et al., 2002a } \\
\text { (Shelton, 2004) }\end{array}$ & $483 \mathrm{NE}$ & Venlafaxina (NE) & NE & 12 & Venlafaxina/olanzapina (NR) & $\begin{array}{l}\text { Venlafaxina, } \\
\text { olanzapina, } \\
\text { nortriptilina }\end{array}$ & NS \\
\hline Shelton et al., 2005 & 500 UP & 25-50mg fluoxetina & $6-12$ & 8 & $\begin{array}{c}\text { Fluoxetina/olanzapina } \\
146 / 40(27,5)\end{array}$ & $\begin{array}{c}\text { Fluoxetina } \\
\text { 142/41 }(28,9), \\
\text { olanzapina } \\
\text { 144/28 }(19,3) \\
\text { nortriptilina } \\
68 / 21(30,3)\end{array}$ & $\begin{array}{c}\text { NS (end point) } \\
(<0,05 \text { em } \\
\text { análise pos } \\
\text { hoc) }\end{array}$ \\
\hline Corya et al., 2006 & 483 & $\begin{array}{l}\text { Fluoxetina (NE), } \\
\text { venlafaxina (NE) }\end{array}$ & NE & 12 & Fluoxetina/olanzapina & $\begin{array}{l}\text { Fluoxetina, } \\
\text { olanzapina, } \\
\text { venlafaxina }\end{array}$ & $\begin{array}{c}\text { Vs. olanzapina } \\
(<0,001) \text {, } \\
\text { vs. fluoxetina } \\
(0,006) \\
\text { vs. venlafaxina } \\
(0,98)\end{array}$ \\
\hline Total & 1.530 & & & & & & \\
\hline
\end{tabular}

NS: não-significativo; UP: depressão unipolar; BP: história de bipolaridade; NE: não-especificado. 
sivos em pacientes com DRT unipolar. A buspirona também foi pouco estudada, e os estudos existentes não sugerem que possa ter papel relevante em DRT. Os estudos duplo-cegos sobre o pindolol como potencializador de antidepressivos são escassos e os resultados, controversos, não permitindo concluir sobre sua eficácia na DRT. A carbamazepina foi muito pouco estudada em DRT. A lamotrigina ainda não foi adequadamente avaliada na potencialização de antidepressivos em pacientes com DRT, e no estudo controlado existente observou-se evidência menor de eficácia derivada da análise de critério secundário de avaliação de eficácia.

O lítio foi o preditor de resposta terapêutica mais estudado, embora ainda existam controvérsias quanto a interferência da gravidade da depressão, cronicidade, número de episódios prévios, presença de co-morbidades, história de bipolaridade, tempo de depressão e grau de resistência nos efeitos terapêuticos. Além disso, seu mecanismo de ação, o tempo necessário para início da resposta, a possibilidade de relação entre resposta e doses dos antidepressivos, a determinação de seus níveis plasmáticos eficazes, a duração do tratamento, a eficácia em determinados subtipos de depressão, quando optar por seu uso na DRT, e a eficácia na potencialização de outros antidepressivos permanecem sem resposta. A buspirona, o pindolol, a carbamazepina, a lamotrigina e a olanzapina não foram estudados adequadamente a esse respeito.

As doses recomendadas são as doses terapêuticas usuais para o lítio; $25 \mu \mathrm{g}$ para o hormônio tireoidiano; 20 a 30mg para a buspirona; $2,5 \mathrm{mg}$ três vezes ao dia para o pindolol; e 200mg para a lamotrigina. A dose de carbamazepina utilizada no único estudo controlado de potencialização variou de 400 a $800 \mathrm{mg}$; a de olanzapina, de 5 a $20 \mathrm{mg}$ nos diversos ensaios clínicos.

A tolerabilidade e a segurança das estratégias estudadas foram avaliadas. 0 emprego de lítio ou do hormônio tireoidiano requer monitoração da função tireoidiana; no caso da buspirona, devem-se ponderar os riscos de síndrome serotonérgica e interação com antidepressivos que inibem a enzima $3 \mathrm{~A} 3 / 4$ do sistema citocromo P450. O pindolol exige cautela na prescrição para pacientes asmáticos, alérgicos graves, portadores de problemas na condução cardíaca e diabéticos. A carbamazepina requer atenção ao risco de interações medicamentosas. Alamotrigina exige titulação cautelosa da dose devido ao risco de síndrome de Stevens-Johnson; exige também atenção ao risco de interação com o valproato. Aolanzapina está associada a ganho de peso e desenvolvimento de síndrome metabólica, demanda orientação nutricional e recomendação de atividade física.

Os estudos sobre DRT apresentaram problemas metodológicos importantes: utilização de diferentes definições de DRT; não-explicitação do critério de definição de resistência utilizado em ensaios (Goodwin et al., 1982; Kramlinger e Post, 1989); utilização de amostras insuficientes (De Montigny et al., 1983; Heninger et al., 1983; Cournoyer et al., 1984; Kantor et al., 1986; Zusky et al., 1988; Schopf et al., 1989; Kramlinger e Post, 1989; Dinan e Barry, 1989; Browne et al., 1990, Stein e Bernardt, 1993; Baumann et al., 1996; Nierenberg et al., 2003; Maes et al., 1996; Moreno et al., 1997; Maes et al., 1999; Sokolski et al., 2004; Barbosa et al., 2003); realização de estudos não-controlados com placebo (Hoencamp, 1994; Fava et al., 1994 e 2002; Maes et al., 1999; Rybakowski et al., 1999; Corya et al., 2006); não-explicitação de inclusão (Maes et al., 1996; Maes et al., 1999; Sokolski et al., 2004), ou inclusão e análise em conjunto, de pacientes com história de episódio(s) de mania ou de hipomania (Heninger et al., 1983; Dinan e Barry, 1989; Schopf et al., 1989; Hoencamp et al., 1994; Katona et al., 1995; Baumann et al., 1996; Stein e Bernardt, 1993; Normann et al., 2002; Barbosa et al., 2003; Dubé et al., 2002a; Dubé et al., 2002b); não-informação sobre o tempo de uso prévio do antidepressivo (Steiner et al., 1978; Fliers, 2003; Maes et al., 1996; Maes et al., 1999; Dubé et al., 2002a, Dubé et al., 2002b); inclusão de pacientes que não fizeram uso do antidepressivo pelo período de tempo mínimo preconizado (Heninger et al., 1983; De Montigny et al., 1983; Kantor et al., 1986; Aronson et al., 1996). Além disso, alguns fármacos, como a buspirona, o pindolol, a carbamazepina e a lamotrigina, foram pouco estudados e outros, como a risperidona, a ziprasidona, a clozapina e a quetiapina, não foram estudados em ensaios duplo-cegos.

Esses aspectos metodológicos contribuíram para a elaboração de algoritmos e consensos terapêuticos (Moreno e Moreno, 1993; Bauer e Dopfmer, 1999; Bauer et al., 2003; Stimpson et al., 2002). Ademais, existem lacunas no conhecimento neurobiológico (Ananth, 1998) acerca dos transtornos psiquiátricos e seu tratamento, o que constitui fator ainda mais limitante, pois elas independem de questões metodológicas, já mais bem esclarecidas. A decisão na escolha de uma estratégia de potencialização - e não de outra - deverá atender, então, às evidências de eficácia, às características do paciente, ao seu estado clínico, à tolerabilidade e à segurança de cada fármaco (Ananth, 1998; Bailey, 2003).

\section{Referências}

Ananth J. Treatment-resistant depression. Psychother Psychosom, 67: 61 70, 1998.

Appelberg BG, Syvalahti EK, Koskinen TE, Mehtonen OP, Muhonen TT, Naukkarinen $\mathrm{HH}$. Patients with severe depression may benefit from buspirone augmentation of selective serotonin reuptake inhibitors: results from a placebo-controlled randomized, double-blind, placebo wash-in study. J Clin Psychiatry, 62: 448-52, 2001.

Aronson R, Offman HJ, Joffe RT, Naylor D. Triiodothyronine augmentation in the treatment of refractory depression. Arch Gen Psychiatry, 53: 842-8, 1996.

Artigas F, Perez V, Alvarez E. Pindolol induces a rapid improvement of depressed patients treated with serotonin reuptake inhibitors. Arch Gen Psychiatry, 51: 248-9, 1994.

Baetz M, Malcolm D. Serotonin syndrome from fluvoxamine and buspirone. Can J Psychiatry, 40: 428-9, 1995.

Bailey KP. Treating treatment-resistant depression: whether to switch, augment, or combine therapies. J Psychosoc Nurs Ment Health Serv, 41(6): 1420, 2003.

Bakish D. Fluoxetine potentiation by buspirone: three case histories. Can J Psychiatry, 36: 749-50, 1991.

Barbee JG, Jamhour NJ. Lamotrigine as an augmentor agent in treatmentresistant depression. J Clin Psychiatry, 63: 737-41, 2002. 
Barbosa L, Berk M, Vorster M. A double-blind, randomized, placebocontrolled trial of augmentation with lamotrigine or placebo in patients concomitantly treated with fluoxetine for resistant major depressive episodes. J Clin Psychiatry, 64: 403-7, 2003.

Bauer M, Dopfmer S. Lithium augmentation in treatment-resistant depression: meta analysis of placebo-controlled studies. J Clin Psychopharmacol, 19: 427-34, 1999.

Bauer M, Adli M, Baethge C, Berghofer A, Sasse J, Heinz A, Bschor T. Lithium augmentation therapy in refractory depression: clinical evidence and neurobiological mechanisms. Can J Psychiatry, 7: 440-8, 2003.

Baumann P, Nil R, Souche A, Montaldi S, Baettig D, Lambert S, Uehlinger C, Kasas A, Amey M, Jonzier-Perey A. A double-blind, placebocontrolled study of citalopram with and without lithium in the treatment of therapy-resistant depressive patients: a clinical, pharmacokinetic and pharmachogenetic investigation. J Clin Psychopharmacol, 16 (4): $307-14,1996$.

Blier P, Bergeron R. The use of pindolol to potentiate antidepressant medication. J Clin Psychiatry, 59(Suppl 5): 16-23, 1998.

Bowden CL, Calabrese JR, Sachs G, Yatham LN, Ashghar SA, Hompland M, Montgomery P, Earl N, Smoot TM, DeVeaugh-Geiss J. A placebocontrolled 18-month trial lamotrigine and lithium maintenance treatment in recently manic or hypomanic patients with bipolar I disorder. Arch Gen Psychiatry, 60: 392-400, 2003.

Browne M, Lapierre, YD, Hrdina PD, Horn E. Lithium as an adjunct in the treatment of major depression. Int Clin Psychopharmacol, 5: 103-10, 1990

Bschor T, Canata B, Müller-Oerlinghausen B, Bauer M. Predictors of response to lithium augmentation in tricyclic antidepressant-resistant depression. J Affect Disord, 64(2-3): 261-5, 2001.

Calabrese JR, Bowden CL, McElroy SL, Cookson J, Andersen J, Keck PE, Rhodes L, Bolden-Watson C, Zhou J, Ascher JA. Spectrum of activity of lamotrigine in treatment-refractory bipolar disorder. Am J Psychiatry, 156(7): 1019-23, 1999.

Calabrese JR, Rapport DJ, Shelton MD, Kimmel SE. Clinical studies on the use of lamotrigine in bipolar disorder. Neuropsychobiol, 38: 185-91, 1998

Chopra P, Schweitzer I. Serotonine syndrome associated with fluoxetine and olanzapine. World Biol Psychiatry, 2: 114-5, 2003.

Corya SA, Andersen SW, Detke HC, Kelly LS, Van Campen LE, Sanger TM, Williamson DJ, Dubé S. Long-term antidepressant efficacy and safety of olanzapine/fluoxetine combination: a 76-week open-label study. J Clin Psychiatry, 11: 1349-56, 2003.

Corya SA,Williamson DJ, Sanger TM, Briggs SD, Case M, Tollefson G. A randomized, double-blind comparison of olanzapine/fluoxetine combination, olanzapine, fluoxetine, and venlafaxine in treatmentresistant depression. Depress Anxiety, 23(6): 364-72, 2006.

Cournoyer G, Montigny $\mathrm{C}$, Ouellette $\mathrm{J}$ et al. Lithium addition in tricyclic resistant unipolar depression: a placebo-controlled study. Int J Neuropsychopharmacol, 14: 17, 1984.

De Montigny C, Cournoyer G, Morrisset R, Langlois R, Caillé G. Lithium carbonate addition in tricyclic antidepressant-resistant unipolar depression. Arch Gen Psychiatry, 40: 1327-34, 1983.

Dietrich DE, Emrich $\mathrm{H}$. The use of anticonvulsants to augment antidepressant medication. J Clin Psychiatry, 59(Suppl 5): 51-9, 1998.

Dimitriou EC, Dimitriou CE. Buspirone augmentation of antidepressant therapy. J Clin Psychopharmacol, 6: 465-9, 1998.

Dinan TG, Barry S. A comparison of eletroconvulsive therapy with a combined lithium and tricyclic combination among depressed tricyclic non-responders. Acta Psychiatr Scand, 80: 97-100, 1989.

Dubé S, Corya SA, Andersen SW et al. Efficacy of olanzapine-fluoxetine combination in treatment-resistant depression. Apresentado no $41^{\circ}$ Encontro Annual do Colégio Americano de Neuropsicofarmacologia. San Juan, Porto Rico; 8-9 dez, 2002a.
Dubé S, Paul S, Sanger T et al. Olanzapine-fluoxetine combination in treatment-resistant depression. Eur Psychiatry, 17(Suppl 1): 98 , 2002b.

Fava M, Alpert J, Nierenberg A, Lagomasino I, Sonawalla S, Tedlow J, Worthington J, Baer L, Rosenbaum JF. Double-blind study of high-dose fluoxetine versus lithium or desipramine augmentation of fluoxetine in partial responders and non-responders to fluoxetine. $J$ Clin Psychopharmacol, 22: 379-87, 2002.

Fava M, Davidson KG. Definition and epidemiology of treatment-resistant depression. Psychiatr Clin North Am, 19: 179-99, 1996.

Fava M, Rosenbaum JF, McGrath PJ, Stewart JW, Amsterdan JD, Quitkin FM. Lithium and tricyclic augmentation of fluoxetine treatment for resistant major depression: a double-blind, controlled study. Am J Psychiatry, 151: 1372-4, 1994.

Fava M. Augmentation and combination strategies in treatment-resistant depression. J Clin Psychiatry, 62(Suppl 18): 4-11, 2001.

Fava M. New approaches to the treatment of refractory depression. J Clin Psychiatry, 61(Suppl 1): 26-32, 2000.

Fisher $\mathrm{P}$, Tauscher J, Küfferle B, Kasper S. Weak antidepressant response after buspirone augmentation of serotonin reuptake inhibitors in refractory severe depression. Int Clin Psychopharmacol, 13: 83-6, 1998.

Fliers E. T3 fails to augment antidepressant effects. $85^{\text {th }}$ Annual Meeting of the Endocrine Society: Philadelphia, Pennsylvania, 2003.

Gitlin MJ, Weiner H, Fairbanks L, Hershmean JM, Friedfeld N. Failure of T3 to potentiate tricyclic antidepressant response. J Affect Disord, 13: 267-72, 1987.

Goodwin FK, Prange AJ, Post RM, Muscettola G, Lipton MA. Potentiation of antidepressant effects by L-triiodothyronine in tricyclic nonresponders. Am J Psychiatry, 1: 34-8, 1982.

Greenberg P, Corey-Lisle PK, Birnbaum H, Marynchenko M, Claxton A. Economic implications of treatment-resistant depression among employees. Pharmacoeconomics, 6: 364-73, 2004.

Hamilton M. A rating scale for depression. J Neurol Neurosurg Psychiatry, 23: 56-62, 1960.

Heninger GR, Charney DS, Sternberg DE. Lithium carbonate augmentation of antidepressant treatment: an effective prescription for treatmentrefractory depression. Arch Gen Psychiatry, 40: 1335-42, 1983.

Hoencamp E, Haffmans PMJ, Dijken WA, Hoogduin CAL, Nolen WA, van Dyck R. Brofaromine versus lithium addition to maprotiline: a doubleblind study in maprotiline-refractory depressed outpatients. J Affect Disorders, 30(3): 219-27, 1994.

Jacobsen M. Possible augmentation of antidepressant response by buspirone. J Clin Psychiatry, 5: 217-20, 1991.

Jefferson JW. Lamotrigine in psychiatry: pharmacology and therapeutics. CNS 2005, 10: 224-32.

Joffe RT, Singer W. A comparison of triiodothyronine and thyroxine in the potentiation of tricyclic antidepressants. Psychiatry Research, 32: 241-51, 1990.

Joffe RT, Schuller DR. An open study of buspirone augmentation of serotonin reuptake inhibitors in refractory depression. J Clin Psychiatry, 54, 7: 269-71, 1993

Joffe RT, Singer W, LevittAJ, MacDonald C. A placebo-controlled comparison of lithium and triidothyronine augmentation of tricyclic antidepressants in unipolar refractory depression. Arch Gen Psychiatry, 50: 87-93, 1993a.

Joffe RT, LevittAJ, Bagby RM, MacDonald C, Singer W. Predictors of response to lithium and triidodothyronine augmentation of antidepressants in tricyclic non-responders. Br J Psychiatry, 163: 574-8, 1993b.

Joffe RT. The use of thyroid supplements to augment antidepressant medication. J Clin Psychiatry, 59(Suppl 5): 26-9, 1998.

Kantor D, McNevin Stephen, Leichner P, Harper D, Krenn M. The benefit of lithium carbonate adjunct in refractory depression - fact of fiction? Can J Psychiatry, 31: 416-8, 1986. 
Katona ALE, Abou-Salen MT, Harrison DA, Nairac BA, Edwards DRL, Lock T, Burns RA, Robertson MM. Placebo-controlled trial of lithium augmentation of fluoxetine and lofepramine. Brit J Psychiatry, 166: 80-6, 1995.

Kramlinger KG, Post RM. The addition of lithium to carbamazepine: antidepressants efficacy in treatment-resistant depression. Arch Gen Psychiatry, 46: 794-800, 1989.

Landén M, Bjorling G, Agren H, Fahlén T. A randomized, double-blind, placebo-controlled trial with buspirone in combination with a SSR in patients with treatment-refractory depression. J Psychiatry 59,12 : 664-8, 1998.

Maes M, Vandoolaeghe E, Desnyder R. Efficacy of treatment with trazodone in combination with pindolol or fluoxetine in major depression. $\mathrm{J}$ Affect Disorders, 41: 201-10, 1996.

Maes M, Libbrech I, vans Hunsel F, Campens D, Meltzer H. Pindolol and mianserin augment the antidepressant activity of fluoxetine in hospitalized major depressed patients, including those with treatment resistance. J Clin Psychopharmacol, 19: 177-82, 1999.

Maltese TM. Adjunctive lamotrigine treatment for major depression [letter] Am J Psychiatry, 156: 1833, 1999.

Montgomery SA, Asberg M. A new depression scale designed to be sensitive to change. Br J Psychiatry, 134: 382-9, 1979.

Moreno AF, Gelenberg AJ, Bachar K, Delgado P. Pindolol augmentation of treatment-resistant depressed patients. J Clin Psychiatry, 58: 437-9, 1997.

Moreno DH, Moreno RA. Depressões resistentes a tratamento: proposta de abordagem. J Bras Psiq, 42(Suppl 10): 41S-5S, 1993.

Nasrallah HA, Newcomer JW. Atypical antipsychotics and metabolic dysregulation: evaluating the risk/benefit and improving the standard of care. J Clin Psychopharmacol, 24(Suppl 1): 7-14, 2004.

Nelson CJ. Managing treatment-resistant major depression. J Clin Psychiatry, 64(Suppl 1): 5-12, 2003.

Nierenberg AA, Price LH, Charney DS, Heninger GR. After lithium augmentation: a retrospective follow-up of patients with antidepressantrefractory depression. J Affect Disord, 18 (3): 167-75, 1990.

Nierenberg AA, Papakostas GI, Petersen T, Montoya HD, Worthington JJ, Tedlow J, Alpert JE, Fava M. Lithium augmentation of nortriptyline for subjects resistant to multiple antidepressants. J Clin Psychopharmacol, 23: 92-5, 2003.

Normann C, Hummel B, Lars OS, Hörn M, Grunze H, Walden J. Lamotrigine as adjunctive to paroxetine in acute depression: a placebo double-blind study. J Clin Psychiatry, 63(4): 337-44, 2002. J Clin Psychiatry, 64(4): 403-7, 2003

Ostroff RB, Nelson C. Risperidone augmentation of selective serotonine reuptake inhibitors in major depression. J Clin Psychiatry, 4: 256-9, 1999.

Papakostas GI, Petersen TJ, Nierenberg AA, Murakami JL, Alpert JE, Rosenbaum JF, Fava M. Ziprasidone: augmentation of selective serotonine reuptake inhibitors (SSRIs) for SSRI-resistant major depressive disorder. J Clin Psychiatry, 2: 217-21, 2004.

Papakostas GI, Petersen TJ, Kinrys G, Burns AM, Worthington JJ, Alpert JE, Fava M, Nierenberg AA. Aripiprazole augmentation of selective serotonin reuptake inhibitors for treatment-resistant major depressive disorder. J Clin Psychiatry, 66(10): 1326-30, 2005.

Perez V, Soler J, Puigdemont D et al. A double-blind, randomized, placebocontrolled trial of pindolol augmentation in depressive patients resistant to serotonine reuptake inhibitors. Arch Gen Psychiatry, 56: 375-9, 1999.

Perlis RH, Nierenberg AA, Mischoulon D, Yeung A, Rosenbaum JF, Fava M. Clinical and sociodemographic predictors of response to augmentation, or dose increase among depressed outpatients resistant to fluoxetine 20mg/day. Acta Psychiatr Scand, 108: 432-8, 2003.

Perry EB, Berman RM, Sanacora G, Anand A, Lynch-Colonese K, Charney DS. Pindolol augmentation in depressed patients resistant to selective serotonine reuptake inhibitors: a double-blind, randomized, controlled trial. J Clin Psychiatry, 65: 238-43, 2004.

Post RM, Denicoff KD, Frye MA, Dunn RT, Leverich GS, Osuch E, Speer A. A history of the use of anticonvulsants as mood stabilizers in the last two decades of the 20th century. Neuropsychobiol, 38: 152-66, 1998.

Rocha FL, Hara C. Lamotrigine as an augmentation agent in treatmentresistant depression. Int Clin Psychopharmacol, 18: 97-9, 2003.

Rocha FL, Hara C. Interações medicamentosas: antidepressivos novos e o sistema citocromo P450. J Bras Psiq, 47(1): 9-18, 1998.

Rouillon F, Gorwood P. The use of lithium to augment antidepressant medication. J Clin Psychiatry, 59(Suppl 5): 32-41, 1998.

Rybakowski JK, Suwalska A, Chlopocka-Wozniak M. Potentiation of antidepressants with lithium or carbamazepine in treatment-resistant depression. Neuropsychobiol, 40: 134-9, 1999.

Schopf J, Baumann P, Rey M. Treatment of endogenous depressions resistant to tricyclic antidepressants or related drugs by lithium addition. Results of a placebo-controlled double-blind study. Pharmacopsychiatry, 22(5): 183-7, 1989

Shelton R, Tllerfson G, Tohen M, Stahl S, Kimberley G, Jacos T, Buras W, Bymaster F, Zhang W, Spencer K, Feldman P, Meltzer H. A nove augmentation strategy for testing treatment-resistant major depression. Am J Psychiatry, 158(1): 131-4, 2001

Shelton RC. Novel antipsycotics for treatment-resistant depression. Psychiatric Times, 21(11), 2004

Shelton RC, Williamson DJ, Corya SA, Sanger TM, Van Campen LE, Case M, Briggs SD, Tollefson GD. Olanzapine/fluoxetine combination for treatment-resistant depression: a controlled study of SSRI and notriptyline resistance. J Clin Psychiatry, 66(10): 1289-97, 2005.

Simon JS, Nemeroff CB. Aripiprazol augmentation of antidepressants for the treatment of partially responding and nonresponding patients with major depressive disorder. J Clin Psychiatry, 66(10): 1216-20, 2005.

Sokolski KN, Conney JC, Brown BJ, De Met EM. Once-daily high-dose pindolo for SSRI-refractory depression. Psychiatry Res, 125: 81-6, 2004.

Spigset O, Adielsson G. Combined serotonin syndrome and hyponatraemia caused by citalopram-buspirone interection. Int Clin Psychopharmacol, 12: 61-3, 1997

Stein G, Bernardt M. Lithium therapy in tricyclic-resistant depression: a controlled trial using lithium in low and normal doses. Brit J Psychiatry, 162: 634-40, 1993

Steiner M, Radwan M, Elizur A, Blum I, Atsmon A, Davidson S. Failure of L-triidothyronine (T3) to potentiate tricyclic antidepressant response. Curr Ther Res Clin Exp, 23: 655-9, 1978.

Stimpson N, Agrawal N, Lewis G. Randomised controlled trials investigating pharmacological and psychological interventions for treatment-refractory depression: systematic review. Brit J Psychiatry, 181: 284-94, 2002.

Stoll AL, Haura G. Tranylcypromine plus risperidone for treatment-refractory major depression. J Clin Psychopharmacol, 4: 495-6, 2000.

Sussman N. Anxiolytic antidepressant augmentation. J Clin Psychiatry, 59(Suppl 5): 42-8, 1998.

Sussman N, Joffe RT. Antidepressant augmentation: conclusions and recommendations. J Clin Psychiatry, 59(Suppl 5): 70-3, 1998.

Tani K, Takei N, Kawai M, Suzuki K, Sekine Y, Toyoda T, Minabe Y, Mori $\mathrm{N}$. Augmentation of milnacipran by risperidone in treatment for major depression. Int J Neuropsychopharmacol, 7: 55-8, 2004.

Thase ME, Howland RH, Friedman ES. Treating antidepressant nonresponders with augmentation strategies: an overview. J Clin Psychiatry, 59(Suppl 5): 5-12, 1998

Thase ME. Therapeutic alternatives for difficult-to-treat depression: a narrative review of the state of the evidence. CNS Spectrums, 11 808-21, 2004

Zusky PM, Biederman J, Rosenbaum JF, Manschreck TC, Gross CC Weilberg JB, Gastfriend DR. Adjunct low-dose lithium carbonate in treatment-resistant depression: a placebo-controlled study. J Clin Psychopharmacol, 8: 120-4, 1988. 